\title{
Mixing, enhanced helium and blue tails in globular clusters
}

\author{
V. Caloi ${ }^{1}$ \\ Istituto di Astrofisica Spaziale C.N.R., Via Fosso del Cavaliere, 00133 Roma, Italy
}

Received 14 September 2000 / Accepted 6 November 2000

\begin{abstract}
We investigate the consequences of an increase in the envelope helium abundance of pre-helium flash red giants in globular clusters, an occurrence suggested by chemical peculiarities in many red giant atmospheres. Comparing predictions with the CM diagrams of a few crucial globular clusters, one finds no evidence for a substantial increase in the surface helium content of horizontal branch members of these clusters, at least for objects in the RR Lyrae region or close to it. The possibility that the most peculiar giants belong to the asymptotic giant branch is discussed. The consequences of a delay in the helium flash are briefly examined.
\end{abstract}

Key words. stars: horizontal branch - stars: AGB and post-AGB - globular clusters: general - stars: chemically peculiar

\section{Introduction}

The evolutionary phase so called of the "horizontal branch" (HB) for Population II stars is well known to depend strongly on the conditions inherited from the evolution along the giant branch (GB). Phenomena that do not alter the overall appearance of the GB on the CM diagram may influence substantially the morphology of the HB. Mass loss is probably the most important of these events, to which the possibility of noticeable changes in the chemical composition has recently been added.

In the following we shall consider some of the consequences that deviations from standard GB evolution may have on HB structures. In particular, we shall examine the effect of enhanced helium content in HB structures and of a delay of the helium flash at the GB tip. Both questions are relevant for the "second parameter" problem in globular clusters; the former has been investigated recently by many authors (see, f.e., the review by Kraft 1994).

\section{HB models with enhanced helium in the envelope}

The high precision of recent spectroscopic observations of globular cluster red giant stars allowed to confirm the existence of objects with peculiar chemical abundances not expected from current evolutionary models - and to better define the percentages of peculiar atmospheres and the degree of the peculiarity for single objects (see the

Send offprint requests to: V. Caloi,

e-mail: caloi@saturn.ias.rm.cnr.it reviews by Smith 1987; Suntzeff 1993; Kraft 1994). The phenomenon has been interpreted in terms of "deep" mixing, that is, of the mixing of envelope material well into the hydrogen burning shell, in regions where proton captures take place (Denisenkov \& Denisenkova 1990; Langer \& Hoffman 1995; Cavallo et al. 1996; Pilachowski et al. 1996; Shetrone 1996).

One of the consequences of this complex phenomenon should be the increase of the helium abundance in the envelope of a star that suffers episodes of deep mixing (Langer \& Hoffman 1995). Such an increase seems assured when $\mathrm{Al}$ is produced at the expense of $\mathrm{Mg}$, but Langer et al. (1997) argue that helium is enhanced also when O and Ne are substantially depleted.

Sweigart (1997) has examined the impact of helium mixing on the morphology of the HB. The process of helium enrichment in the envelope gives rise to an increase in the tip luminosity attained by the red giant star, and so to an increase in the mass lost during the giant branch ascent. Besides, an enhanced helium in the envelope means a bluer HB position at a given total mass (the helium core mass remains almost unchanged).

In Fig. 1 tracks with $Z=0.001$ and three values of the amount of helium in the envelope $(Y=0.245$, 0.285 and 0.345 ) are shown (code ATON2.0, see Ventura et al. 1998; transformations from the theoretical plane through Kurucz 1993). The tracks illustrate the main HB phase until central helium $Y_{\mathrm{c}}=0.1$. A single "breathing pulse" (f.e., Castellani et al. 1985) was sometimes encountered when $Y_{\mathrm{c}} \sim 0.01$ (duration about 1-3 Myr). Since the 


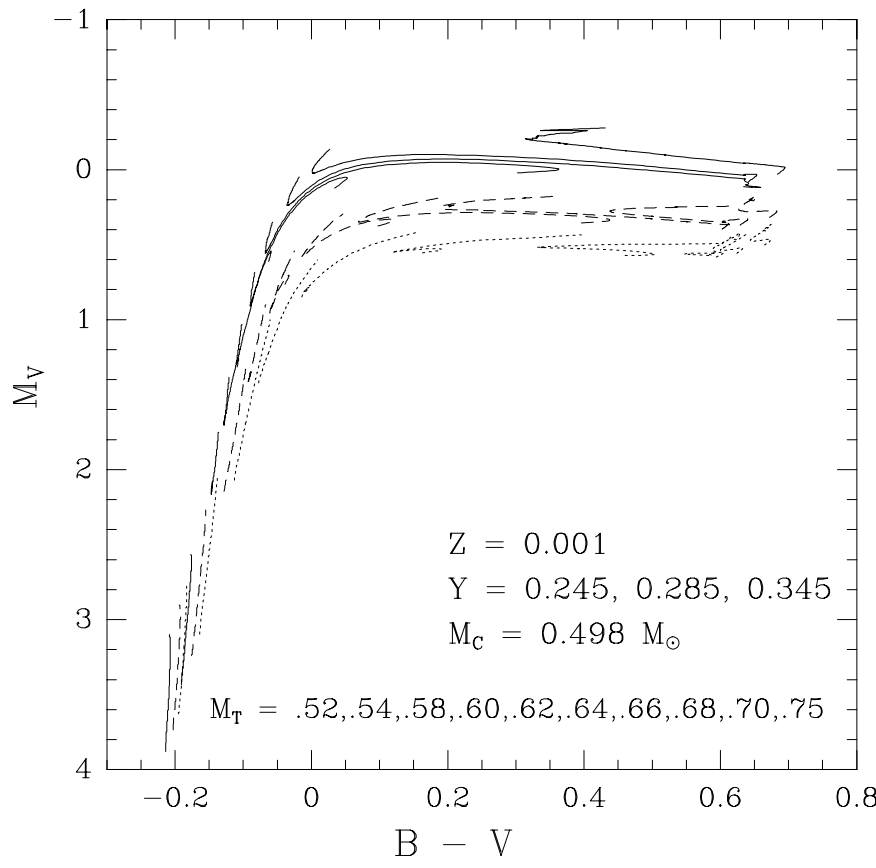

Fig. 1. HB evolutionary tracks of models with the same core mass and envelope $Y=0.245$ (dotted line), 0.285 (dashed line) and 0.345 (solid line). The tracks end when $Y_{\mathrm{c}}=0.1$

phenomenon is generally considered spurious (Dorman \& Rood 1993; Caloi \& Mazzitelli 1993), they have been eliminated from the tracks shown in the figures.

The case $Y=0.245$ is taken to correspond to the normal helium content, after the envelope increase due to the first dredge-up. In Figs. 2 and 3 the tracks are shown for some masses up to the asymptotic giant branch (AGB) for the cases $Y=0.245$ and 0.345 , respectively. The track of a giant of $0.9 M_{\odot}(Z=0.001, Y=0.235)$ is added for reference.

The main evolutionary characteristics appear the following. The mass distribution along the ZAHB does not change substantially with an increase in $Y$ of 0.04 , while the blue loops become longer. The ZAHB luminosity increases of about $0.2 \mathrm{mag}$ (as already noted by Sweigart 1997) and the asymptotic giant branch (AGB) reproduces, at higher luminosity for a given $T_{\text {eff }}$, the one with the lower $Y$ (not shown).

The slower AGB phase begins with a clump due to the ignition of helium in a shell after the end of central burning. For $Y=0.245$ the minimum mass to form the helium shell on the AGB is $0.57 M_{\odot}$; larger masses form the clump more or less at the same position in the CM diagram $\left(M_{v} \sim(-0.3)-(-0.4) \mathrm{mag}\right)$, while lower masses form the helium shell at $B-V \leq 0$ mag and reach the AGB at a luminosity the higher, the lower the mass. Masses $<0.52 M_{\odot}$ do not develop an AGB phase.

The shell formation phase lasts about 1.5-2.5 $10^{6} \mathrm{yr}$ (the lower the mass, the shorter the phase) and another 8-9 $10^{6}$ yr are spent by the star along the AG branch until the first helium shell flash. The luminosity at which

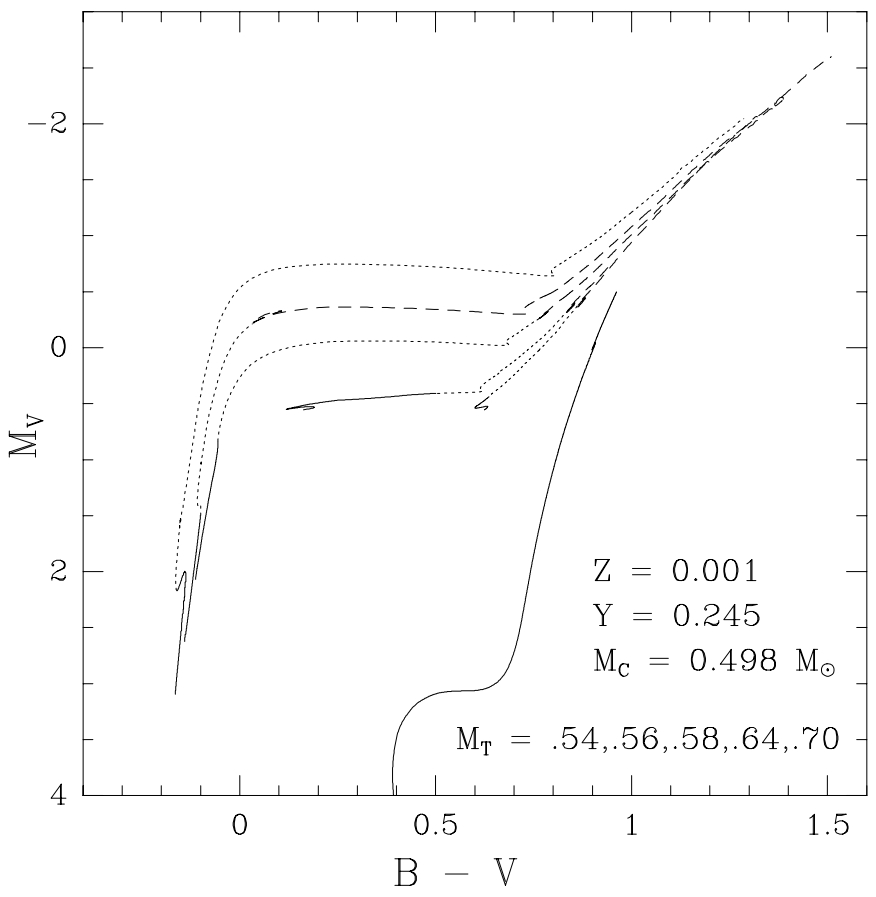

Fig. 2. Evolution out of the HB and up to the AGB for models with envelope $Y=0.245$; dotted lines indicate fast phases, for $M \geq 0.56 M_{\odot}$, dashed lines indicate the helium shell burning phase. The track of a $0.9 M_{\odot}$ red giant is shown as reference

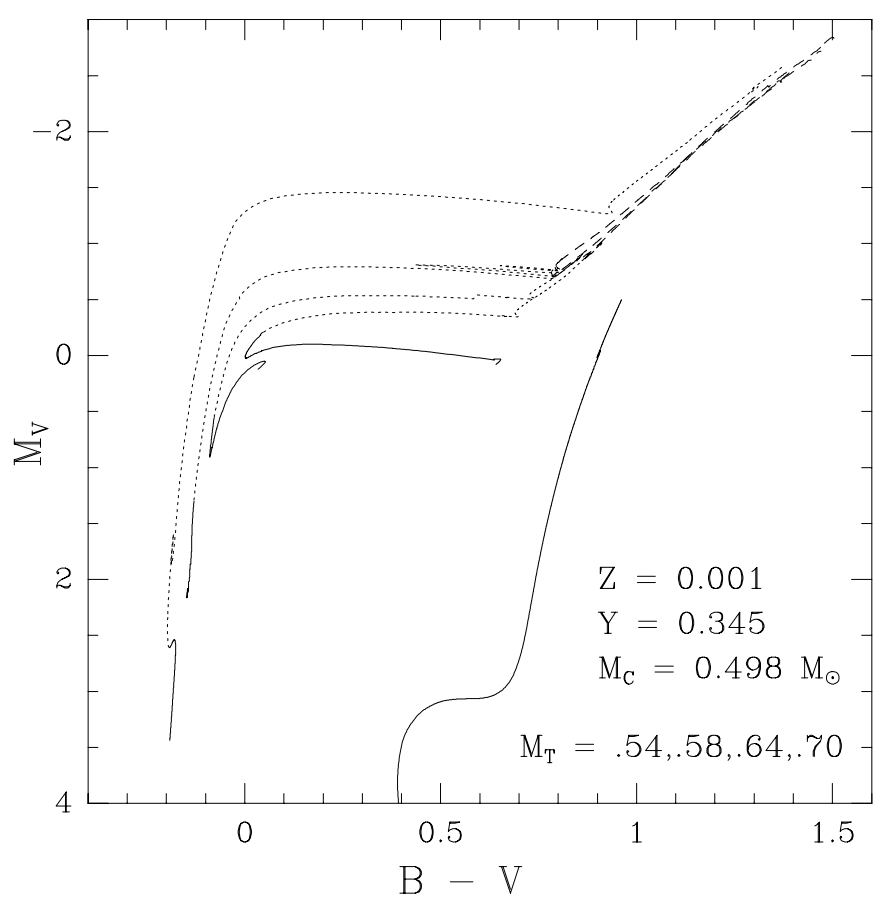

Fig. 3. Evolution out of the HB and up to the AGB for models with envelope $Y=0.345$; dotted lines indicate fast phases, for $M \geq 0.58 M_{\odot}$, dashed lines indicate the helium shell burning phase. The track of a $0.9 M_{\odot}$ red giant is shown as reference

the first flash develops depends slightly on the star mass: it varies from $M_{v} \sim-2.2 \mathrm{mag}\left(\log \left(L / L_{\odot}\right) \sim 3.05\right)$ to 


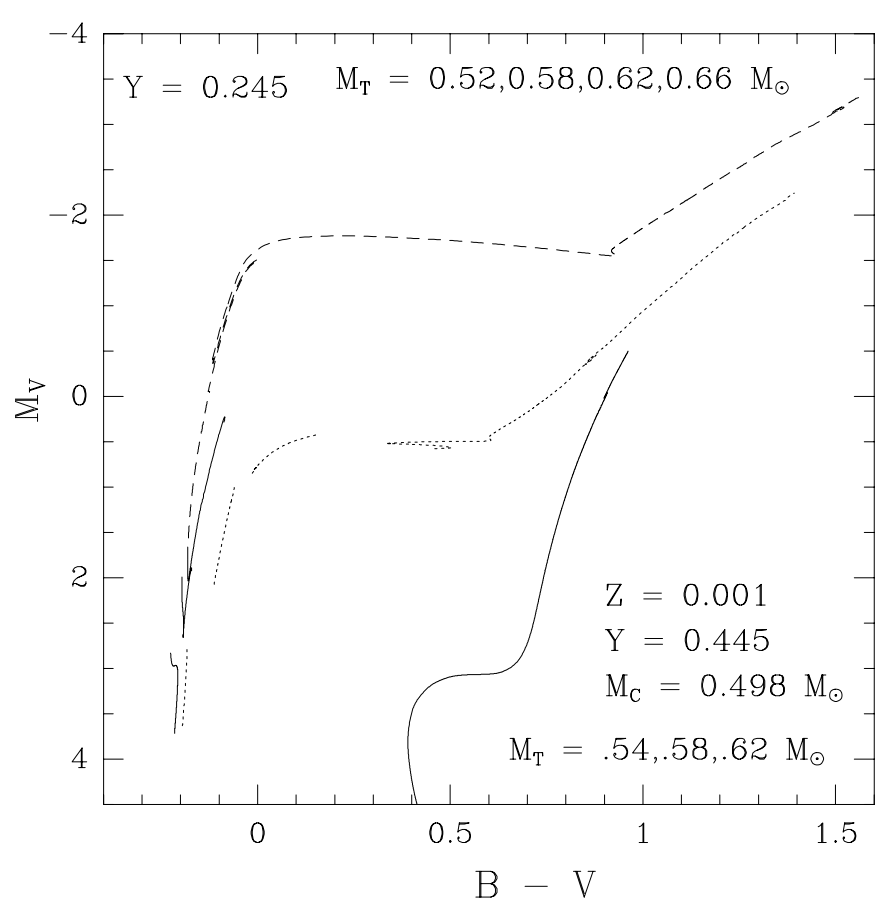

Fig. 4. Evolutionary tracks for HB models with envelope $Y=$ 0.445 ; for $M=0.62 M_{\odot}$ the track is extended to the AGB (dashed line: fast evolution). As reference, HB tracks for $Y=$ 0.245 are shown (dotted line), together with a red giant track for $M=0.9 M_{\odot}($ solid line $)$

$-2.6 \mathrm{mag}\left(\log \left(L / L_{\odot}\right) \sim 3.3\right)$, for an evolving mass of 0.52 and $0.70 M_{\odot}$, respectively.

When compared with the case with $Y=0.245$, horizontal branches with $Y=0.345$ show noticeable differences. The luminosity level at the RR Lyrae gap turns out to be about $0.5 \mathrm{mag}$ more luminous. The tracks of the larger masses show the well known long blue loops (Sweigart \& Gross 1976). The tracks lying in the RR Lyrae domain when $Y=0.245$ move to the blue of the variable gap when $Y$ increases to 0.345 (see the model with $\left.M=0.64 M_{\odot}\right)$.

As for the blue HB, here the effect of the helium content increase is more limited, given the lesser efficiency of the hydrogen shell. The higher molecular weight in the envelope produces higher effective temperatures for a given mass, and so a lower $M_{v}$ due to the larger BCs: so we have that hot structures with larger $Y$ turn out to be less luminous in the visual than their lower helium counterparts (Fig. 1).

The minimum mass for which the shell forms on the $\mathrm{AGB}$ is $0.60 M_{\odot}$, at $M_{v} \simeq-0.6 \mathrm{mag}$ (for comparison, $M=0.7 M_{\odot}$ forms the helium shell at $\left.M_{v} \simeq-0.9 \mathrm{mag}\right)$. The $0.58 M_{\odot}$ model represents a limiting case and forms the helium shell, with some oscillations, at the base of the AGB. Stars with $M \leq 0.53 M_{\odot}$ do not reach the AGB phase. The total time spent on the AGB is always less than $1010^{6} \mathrm{yr}$. On the whole, the AGB with $Y=0.345$ is bluer of $\leq 0.04$ mag than the locus with $Y=0.245$.
We consider an increase in $Y$ of 0.2 only for structures on the blue side of the RR Lyrae gap (Fig. 4). The helium shell forms always at high temperatures and the AGB reduces to a short and luminous phase, well separated from the red giant branch. At the extreme blue edge, a mass of $0.51 M_{\odot}\left(\right.$ envelope mass $\left.=0.012 M_{\odot}\right)$ has a ZAHB magnitude $M_{v}=4.32$, to be compared with a ZAHB magnitude $M_{v}=4.40$ for $0.501 M_{\odot}$ with envelope $Y=0.245$ (envelope mass $=0.003 M_{\odot}$; see Fig. 7).

\section{Comparison with HB populations}

\subsection{The case of $M 3$ and $M 13$}

The clusters M 3 and M 13 offer an interesting possibility of testing the relevance of these departures from standard HB evolution. These clusters have quite different HB populations, notwithstanding the great similarity in the main physical and chemical parameters (see f.e., Catelan \& de Freitas Pachego 1995; Ferraro et al. 1997a). Apparently, they differ only in the ellipticity (M 13 being more elliptical) and in the presence of more rapid rotators in M 13 than in M 3 (Peterson et al. 1995; Behr et al. 2000a). In brief, M 3 has a HB well populated from the region redder than the $\mathrm{RR}$ Lyrae gap to the blue of the gap itself, while M 13 is populated only on the blue side of the gap, with a substantial portion of HB members populating the B subdwarf region.

M 3 and M 13 appear to have the same heavy element content (f.e., Kraft et al. 1992; Carretta \& Gratton 1997). Assuming such a content to be $Z=0.001$ (Carretta \& Gratton 1997), if also the helium content $Y$ is the same in the two clusters, we can estimate that the bulk of $\mathrm{HB}$ objects in M 3 has total mass between 0.68 and $0.62 M_{\odot}$ (see the CM diagrams by Buonanno et al. 1994; Ferraro et al. 1997a,b, and the models by Mazzitelli et al. 1995; Caloi et al. 1997, but any other model set would do). In the same conditions, for M 13 we expect $\mathrm{HB}$ objects from 0.61 to $0.5 M_{\odot}$ (see the CM diagram by Paltrinieri et al. 1998, Fig. 5).

The origin of such a difference is uncertain. The age difference between the two clusters is generally estimated not larger than $1 \mathrm{Gyr}$, but values up to 3 Gyr cannot be excluded (VandenBerg et al 1990; Catelan \& de Freitas Pachego 1995; Stetson et al. 1996; Ferraro et al. 1997a; Johnson \& Bolte 1998): Lee et al. (1994) in fact argued that age is the most important second parameter among globular clusters.

On the other hand, the absence of RR Lyrae variables and the very low mass of the extreme HB members in M 13 (about $0.5 M_{\odot}$ ), suggested by their $M_{v}$ larger than the turn-off magnitude, would require an age difference between M 3 and M 13 of various Gyr (see the discussion in Catelan \& de Freitas Pachego 1995). Alternatively, mass loss along the giant branch would have to differ strongly in the two cases.

In Sweigart's investigation on deep mixing (1997), to each value of helium enhancement correspond an increase 


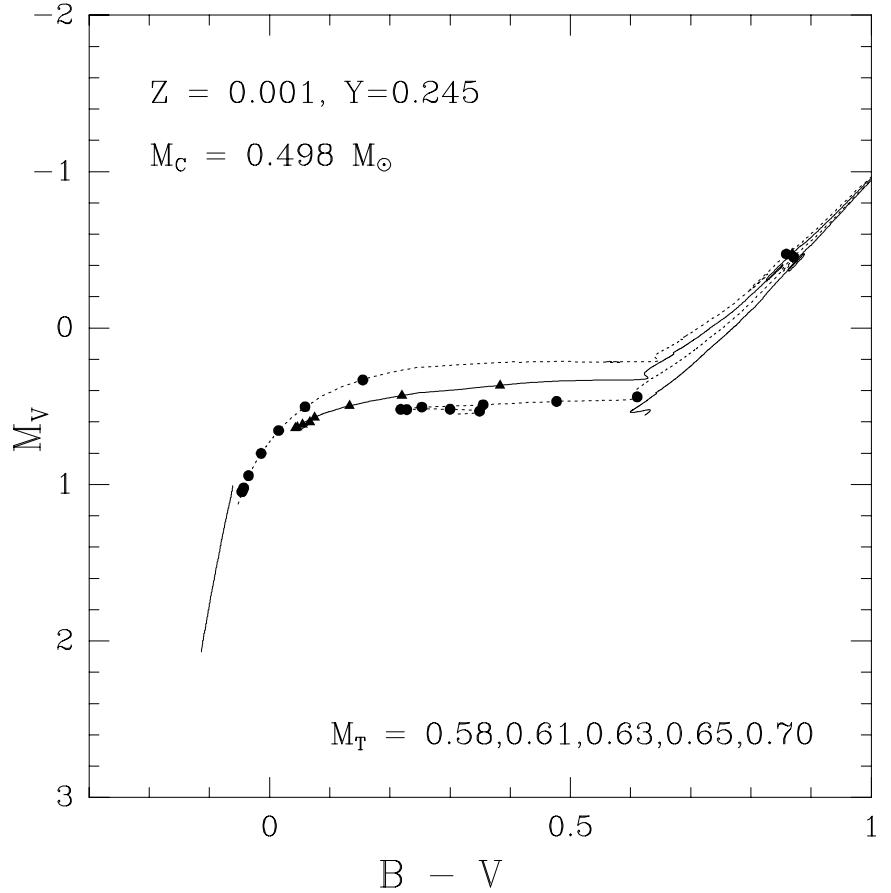

Fig. 5. HB evolutionary tracks illustrating the different mass distributions in M 3 and M 13 once compared with the CM diagrams of the two clusters. Dots and triangles indicate time steps of $10^{7}$

in the GB tip luminosity and an increase in mass loss, while the size of the hydrogen exhausted core remains almost unchanged. It is possible therefore to associate to any position on the standard HB the shift that would result if a certain amount of deep mixing had taken place. Given two globular clusters where deep mixing has occurred at different degrees, it is so possible to predict - at least semi-quantitatively - the difference between the two HB distributions.

To this respect, the relative positions of the horizontal branches in the two clusters are crucial. The main evolutionary loci of the two clusters have been compared by various authors (i.e., Catelan \& de Freitas Pachego 1995; Ferraro et al. 1997a; Johnson \& Bolte 1998). In Fig. 6 we report the data from Johnson \& Bolte (1998) paper, to show that the two CM diagrams $V, V-I$ can be safely superposed (keeping in mind the cautions exemplified in Fig. 7 of the quoted paper).

Similarly, Ferraro et al. (1997a) find that their Hubble Space Telescope CM diagrams $V, U-V$ for $\mathrm{M} 3$ and M 13 overlap nicely, once the HB mean loci are aligned relying on the (small) region where the HB is well populated in both clusters. Only the reddest HB members in M 13 appear more luminous than stars in M 3 at the same colour, and are interpreted by Catelan \& de Freitas Pachego (1995) and Ferraro et al. (1997a) as being more evolved than corresponding stars in M 3.

Estimates of the luminosity level of turn-off and $\mathrm{HB}$ for M 3 and M 13 are given by Buonanno et al. (1994),

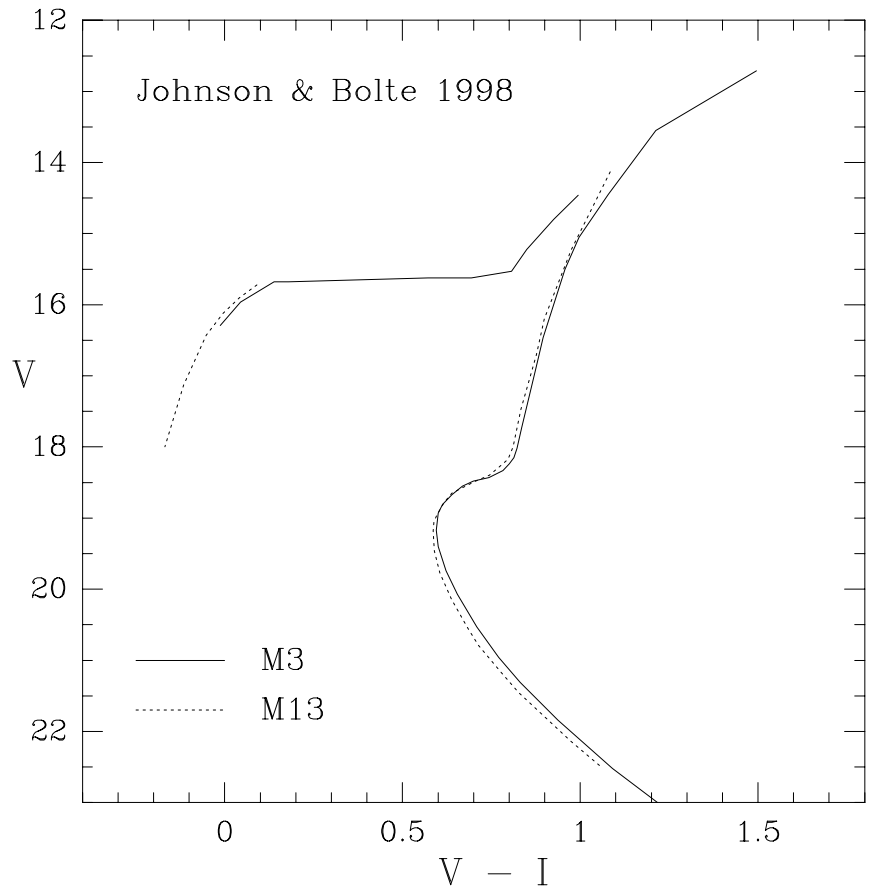

Fig. 6. The observed loci of M 3 and $\mathrm{M} 13$ in the $V, V-I$ plane from Johnson \& Bolte (1998), after a shift in M 13 data of +0.6 mag in $V$

Ferraro et al. (1997b), Paltrinieri et al. (1998). Also these data do not show any noticeable difference in the relative positions of the evolutionary loci in the two clusters.

Therefore, the difference (if any) in luminosity between the HBs in M 3 and M 13 can be safely assumed to be lower than 0.2 mag. This means that the helium enhancement for the coolest M $13 \mathrm{HB}$ members cannot exceed $\delta Y=0.04$ (Sweigart 1997 and Sect. 2 of this paper). The extra mass loss in the GB phase due to this increase in $Y$ in the red giant envelope is of about $0.012 M_{\odot}$ (from Fig. 3 in Sweigart 1997, assuming $\eta=0.4$ and keeping in mind the value for the mixing parameter quoted by the author to give an increase in $Y$ of 0.04 ). A star on the red side of the HB in M 3 has a mass of about $0.68 M_{\odot}$ (for $Z$ about 0.001 , see Fig. 5) and, for the same metallicity, a star at $B-V=0.1$ in M 13 has a mass of about $0.61-0.62$ with the same $Y(0.245)$ or of about $0.64-0.65$ for $Y=0.285$. An extra mass loss of $0.012 M_{\odot}$ appears not sufficient to allow the disappearance of the RR Lyrae variables (see Figs. 1 and 5).

Higher helium mixing and larger mass losses may be present in very blue $\mathrm{HB}$ members $(B-V \leq-0.1)$. In Fig. 7 the effect of an increase $\delta Y=0.2$ in the envelope of extremely blue HB objects is shown. In order to reach an $M_{v} \simeq 4.5 \mathrm{mag}$ (as in M 13 and NGC 6752, where the $\mathrm{HB}$ reaches visual magnitudes higher than the turn-off), a total mass as low as $0.501 M_{\odot}$ is needed for $Y=0.245$, while for $Y=0.445$ a mass $\leq 0.51 M_{\odot}$ is required. So even for extensive mixing one finds that a substantial mass loss is necessary to attain the high magnitudes of extreme HB objects. 


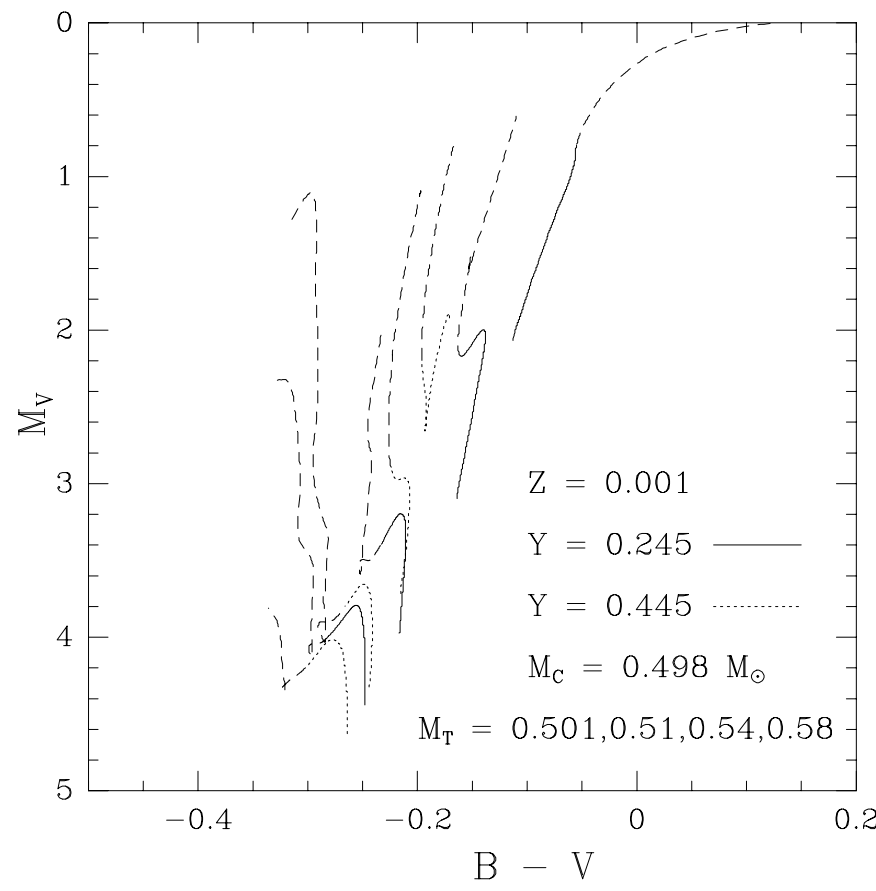

Fig. 7. Evolutionary tracks for HB models with envelope $Y=$ 0.445 (dotted line) and 0.245 (solid line); dashed lines indicate fast phases. With increasing mass the difference in luminosity and colour between models with the same mass increases substantially

Such mass loss turns out larger than expected from the increase in the GB tip luminosity. From Fig. 3 in Sweigart's paper, one gets an extra mass loss of $\sim 0.08 M_{\odot}$ when $\Delta X_{\text {mix }}=0.20(\delta Y \sim 0.2)$; so the minimum mass in M $3\left(\sim 0.62 M_{\odot}\right)$ would become $0.54 M_{\odot}$, definitively larger than the required $0.51 M_{\odot}$ (see Fig. 7).

On the whole, an increase in $Y_{\text {env }}$ is specially efficient in shifting the position on the $\mathrm{HB}$ for the mass range of the RR Lyrae variables and for slightly lower masses (see Fig. 1), but at the price of a noticeable increase in luminosity. Anyway, it is important to keep in mind that this discussion is based on the assumption that mass loss follows strictly Reimer's formulation all along the giant branch. Should mass loss increase more rapidly with luminosity, the conclusions could be different.

\subsection{The case of M 13 and NGC 7006}

Kraft et al. (1998) apply the Sweigart (1997) models to estimate the required amount of helium enhancement in M 13 with respect to NGC 7006, in order to achieve the observed differences in $\mathrm{HB}$ morphology. The required helium abundances are 0.35 and 0.245 for M 13 and NGC 7006, respectively. Figure 1 shows that such abundances require for $\Delta_{\mathrm{ZAHB}}^{\mathrm{TO}}$ in $\mathrm{M} 13$ a value 0.5 mag larger than in NGC 7006, if the two clusters have similar ages (similar turn-off levels). Instead, one finds $3.50 \pm 0.10 \mathrm{mag}$ for both M 13 (see Fig. 6) and NGC 7006 (Buonanno et al. 1991). So it appears difficult to accomodate a difference in $\mathrm{HB}$ level of $0.5 \mathrm{mag}$, without considering an age difference of more than 5 Gyr in the sense of a younger M 13 (since its turn-off would appear more luminous than in NGC 7006).

\subsection{The metal poor clusters}

This difficulty presents itself also in the case of M 15, the metal poor globular cluster with many red giants interested by the signature of deep mixing (Cohen 1979; Sneden et al. 1991; Sneden et al. 1997). Sweigart (1997) observes that an increase in $Y_{\text {env }}$ of 0.04 would give rise to a luminosity difference between the HBs in M 3 and M 15 which would explain the period-shift effect (Sandage 1982). The luminosity increase would be of about $0.2 \mathrm{mag}$.

The comparison with CM diagrams of other very metal poor clusters can give some clues as to the reality of such an increase in $Y_{\text {env }}$. Walker (1994) compared carefully the CM diagrams of $\mathrm{M} 15$ and of M 68, showing that the two diagrams overlap nicely, and that, in particular, the difference in magnitude between $\mathrm{HB}$ and TO $\Delta_{\text {ZAHB }}^{\mathrm{TO}}$ is the same in the two clusters $(3.41 \pm 0.05 \mathrm{mag})$. Similarly, Rey et al. (1998) found for $\Delta_{\mathrm{HB}}^{\mathrm{TO}}$ in M 53 the value of $3.42 \pm 0.05 \mathrm{mag}$. At the same time, the difference in age among the three clusters appear definitively smaller than 2 Gyr.

This indicates that the luminosity of M $15 \mathrm{HB}$ is the same as found in M 68 and M 53. Nothing is known as to peculiar or not peculiar abundances in M 68 and M 53 giants, but they have no blue tail in their HB distributions, and so no hint of extra mass loss on the giant branch or extra helium in their HB structures. Besides, Brocato et al. (1994) and Walker (1994) find that the pulsational properties of M $68 \mathrm{RR}$ Lyrae variables are analogous to those in M 15, suggesting similar luminosities.

It seems therefore that the population on M $15 \mathrm{HB}$ in the region of the RR Lyrae stars resembles quite strictly the population in apparently normal clusters such as M 68 and M 53. The amount of extra helium $\delta Y$ allowed in M 15 structures around the RR Lyrae gap is surely less than 0.04 , which would imply a luminosity increase of $0.2 \mathrm{mag}$ with respect to the HBs in M 68 and M 53. Stars with helium enhanced may be found in bluer positions than the gap surroundings.

Summing up, there are no indications of a substantial increase in the helium content in most of the HB stars in clusters whose giants show evidence of mixing of envelope material into helium rich regions, such as those where $\mathrm{Na}$ and $\mathrm{Al}$ enrichment take place.

\section{Deep mixing: First or asymptotic giant branch?}

The situation with the variety of relative abundances of $\mathrm{CNO}, \mathrm{Na}, \mathrm{Mg}, \mathrm{Al}$, etc. in globular clusters has reached a high degree of complexity (see f.e., the review by Kraft 1994). There is evidence for primordial fluctuations (f.e., $\mathrm{CN}$ versus $\mathrm{CH}$ anticorrelation in main sequence and subgiant stars in NGC 6752, 47 Tuc and M 92: Suntzeff 1989; Bell et al. 1983; Briley et al. 1991; King et al. 1998) as well 
as for evolution induced changes in surface abundances of various atomic species ( $\mathrm{CNO}, \mathrm{Na}, \mathrm{Al}, \mathrm{Mg}$ and their isotopes: quoted paper by Kraft). A large dispersion in $\mathrm{O}$ abundances is even observed in a young cluster in the Small Magellanic Cloud, NGC 330 (Hill 1999).

We would like to consider only one aspect of this vast problem: the evolutionary status of the most affected objects in one of the best studied clusters, M 13. The most luminous giants show strong $\mathrm{O}$ deficiency and $\mathrm{Na}$ overabundance. It is commonly assumed that these giants belong to the first giant branch, on their way to the helium flash.

We suggest on the contrary that a substantial fraction of the objects observed near the giant tip are asymptotic branch stars. This statement is mainly based on the fact that the majority of the luminous giants in M 13 are variables of the semiregular (SR) type with period longer than 30 days, typical of the last phases of the AGB in intermediate and very metal poor globular clusters (f.e., Feast 1999). While Mira variables lie above the red giant tip as defined by the developing of the helium flash, and so we can be sure that they belong to the AGB phase, red SR variables are not always above the RG tip. Nevertheless, it seems likely that they are AGB stars, since: i) there is evidence that in the metal rich globular clusters they evolve into the Mira phase; ii) if we consider the SR variables for which periods can be obtained with some confidence, it appears that the light variations are due primarily to pulsation, as in the case of Miras (Feast 1999); iii) Whitelock (1986) has shown that in globular clusters the luminosities of the SRs increase with the period and that the sequence defined by these stars terminates at the position of the Miras in the cluster (see also Feast 1999).

In Table 1 by Pilachowski et al. (1996), seven out of the ten most luminous giants in M 13 turn out to be variable or probably variable: L324 = V11 in Sawyer Hogg's catalogue (SH 1973; light curve in Osborn \& Fuenmayor 1977), L598 (=B140) probably variable according to Kadla et al. (1976), L194 (= Arp II-90) definitively variable according to Welty (1985), L973 (= Arp I-48) light curve in Osborn \& Fuenmayor (1977), L835 = V15 in SH (light curve in Osborn \& Fuenmayor), L954 (= Arp IV-25) definitively variable according to Welty (1985), L70 (= Arp II-67) probably variable according to Welty. L414 (= Arp III-56) has been suspected, but not confirmed, of being variable (Russev 1973; Osborn \& Fuenmayor 1977).

Five out of the seven tip variables are super-oxygen poor $([\mathrm{O} / \mathrm{Fe}]<-0.4)$ and one is oxygen-poor $([\mathrm{O} / \mathrm{Fe}]=$ -0.26) according to Kraft et al. (1993, Table 6). From Table 1 in Pilachowski et al. (1996) it appears that the $[\mathrm{Na} / \mathrm{Fe}]$ ratios near the red giant branch tip are exclusively high (with the exception of L598), while on the lower giant branch there is a wider range in $\mathrm{Na}$ abundances. We suggets therefore that, whatever the phenomenon involved, it is specially active during the final evolution along the asymptotic giant branch, and in particular in the evolution of stars with small envelopes (as supposedly are the ones found on the AGB of M 13).
The variability of Miras has been associated with the helium shell flashes phase (Feast 1989). Since SR variables appear related to Miras, as mentioned before, also they are likely in the terminal stage of the AGB evolution. We computed a series of 8 flashes for a $0.65 M_{\odot}$ : each flash cycle lasted about $2-310^{5}$ yr and during it the hydrogen exhausted core increased of about $0.009 M_{\odot}$ and the $M_{v}$ at maximum of about $0.09 \mathrm{mag}$. These results have to be taken as indicative, given the well known extremely strong dependence of thermal pulse development on stellar structure details.

As mentioned before (Sect. 2), the flashes begin at increasing luminosty for increasing evolving mass (from $\log \left(L / L_{\odot}\right) \sim 3.05-M_{v} \sim-2.2-$ to $\sim 3.3-M_{v} \sim-2.6-$ for masses from 0.52 to $\left.0.70 M_{\odot}\right)$. These luminosities are in any case very close to the the first GB tip: $\log \left(L / L_{\odot}\right) \sim$ $3.38\left(M_{v} \sim-2.8\right)$ for $Z=0.001$ (f.e., Salaris \& Cassisi 1997). If the luminosity of the AGB termination is close, as generally accepted (Renzini 1977), to the one of the first GB, only a few flashes will take place, for a total duration of the order of $10^{6} \mathrm{yr}$ or less. For comparison, a first GB member moves from $\log \left(L / L_{\odot}\right)=3.05$ to the GB tip in about $310^{6} \mathrm{yr}$, and from 3.3, in $610^{5} \mathrm{yr}$.

So the expected evolutionary rates in luminosity along the two giant branches are similar close to the tip. Actually, since the evolution in luminosity in the AGB pulsing phase is roughly constant (see also Gingold 1974), it may become slower than along the first branch.

In a cluster like M 13, whose HB is populated by stars with mass $\leq 0.62 M_{\odot}$ (see before), most stars will begin their pulsing phase at $M_{v} \sim(-2.2)-(-2.4)$, with a hydrogen exhausted core of about $0.52 M_{\odot}$. An HB mass of $0.58 M_{\odot}$, increasing the core mass of $0.009 M_{\odot}$ per flash, will perform about 6 flashes in absence of mass loss, reaching the luminosity of the first GB tip $\left(M_{v} \sim-2.8\right)$.

In a cluster like M 3, where the most common $\mathrm{HB}$ mass is about $0.65 M_{\odot}$ (the mass in the RR Lyrae gap), the flash phase begins at $M_{v} \sim-2.6$, about $0.2 \mathrm{mag}$ below the theoretical tip and about where we expect to observe the most luminous cluster giants (Frogel 1983; Salaris \& Cassisi 1997, 1998). Without mass loss, the star could perform about 14 pulses, with an increase in luminosity of about $1.3 \mathrm{mag}$, well above the first GB tip. This point hase been discussed by Renzini (1977), who reached the conclusion that during the AGB the evolving mass loses at least $0.1 M_{\odot}$. Just for the sake of the argument, we notice that the most luminous giant in $\mathrm{M} 3$ is a $\mathrm{SR}$ variable (V95 in $\mathrm{SH}=$ vZ318: $V=12.36 \mathrm{mag},(B-V)=1.78 \mathrm{mag}$, period $=103 \mathrm{~d}$, SH and Welty 1985); the second one in luminosity is again a variable (vZ1397 $=\mathrm{SK} 26, V=12.65$, $B-V=1.56 \mathrm{mag}$, period (uncertain) $=60 \mathrm{~d}$, Welty 1985). At about this magnitude, a few more giants are found, so that V95 results isolated $0.3 \mathrm{mag}$ above the bulk of the GB members.

In Sect. 3.1 we saw that M 3 and M 13 CM diagrams can be overlapped with a shift in $V$ of about $0.6 \mathrm{mag}, \mathrm{M} 3$ being the farther away. The tip of M 13 is at $V \sim 12$ mag (Pilachowski et al. 1996), so that we expect the tip of M 3 
at $V=12.6 \mathrm{mag}$, as actually observed, exception made for V95: this may be taken as a consequence of the larger - on the average - AGB masses in M 3 with respect to M 13 (in the limits of small number statistics!).

Some advantages can be found if the mixing takes place in AGB objects. At luminosities close to the first giant branch tip the core mass $M_{\mathrm{c}}$ would be $\sim 0.5 M_{\odot}$, so that an evolving giant of about $0.65 M_{\odot}$ would have an envelope mass of about $0.15 M_{\odot}$, to be mixed with the products of (high temperature) hydrogen shell burning. In the case of an AGB object of the same mass, undergoing helium shell flashes, the envelope mass would be smaller, starting from about $0.13 M_{\odot}$ (in the hypothesis of no mass loss during AGB evolution before the flashes) and rapidly decreasing both for mass loss and for the increase of the hydrogen exhausted core. The abundance changes would be faster and would require the consumption of less hydrogen.

In a cluster like M 13 the evolving AGB masses will be lower than $0.65 M_{\odot}$, so that the envelope masses to be mixed will be definitively smaller than in the first GB case, with an evident advantage for the reshuffling of nuclear species. Just as a curiosity: there are indications that some type II Cepheids exhibit abundance peculiarities, among which possibly helium enhancement, as summarized by Gingold (1985) some years ago. It may be worth while to reconsider the objects in question with modern equipment.

For what concerns the nucleosynthesis beyond the standard CNO cycle, the conditions in the hydrogen shell in AGB structures are not very different from the ones encountered in first GB stars. Peak temperatures are of about $55-5810^{6} \mathrm{~K}$ before the first helium shell flash and slightly higher (58-60) $10^{6} \mathrm{~K}$ during the eighth flash (0.65 $M_{\odot}$, see before). The peaks of CNO energy generation during the flash cycles last about $210^{5} \mathrm{yr}$, sufficient to obtain large sodium and aluminum enhancements in surface abundance (Langer \& Hoffman 1995). A difficulty is given by the estimate by Langer et al. (1997) of a temperature of $7010^{6} \mathrm{~K}$ necessary to explain surface abundances of $\mathrm{Mg}$ isotopes as observed by Shetrone $(1995,1996)$ in the most luminous M 13 giants. As stressed by Langer et al. (1997), the estimate of $7010^{6} \mathrm{~K}$ depends very strongly on these results, for which a confirmation would be welcome for safety. In any case, such a difficulty is present for both the RGB and the AGB hypotheses.

\section{An old conjecture on the origin of extended blue tails}

The evolution along the GB suffers a strong acceleration when approaching the helium flash phase. The rate of mass loss, estimated through Reimer's formula, reaches values of about $510^{-8} M_{\odot} \mathrm{yr}^{-1}(\eta \sim 0.4)$. In the last million year before the flash, models of $0.8-0.9 M_{\odot}(Z \leq 0.001)$ have an increase in core mass of $\sim 0.027 M_{\odot}$ and a decrease in total mass of $0.05 M_{\odot}$. This means that a variation of the timing of the helium flash of half a million year, that is, about $2 \%$ of the time to climb the giant branch from the RG bump luminosity to the tip (30-40 Myr), is sufficient

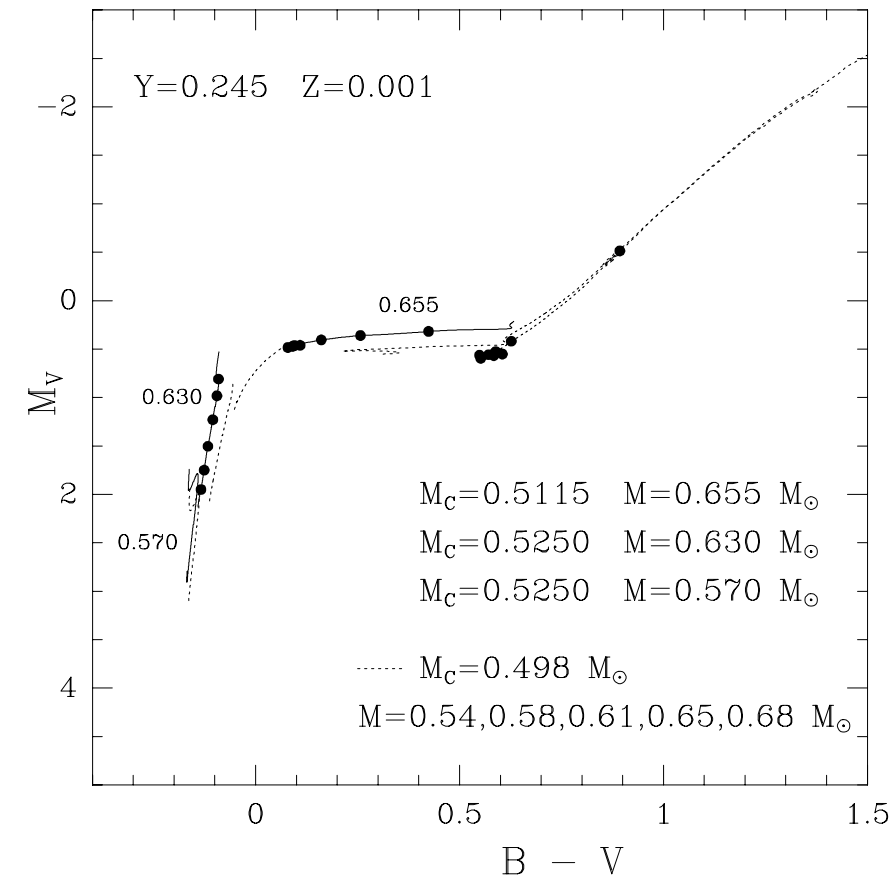

Fig. 8. Evolutionary tracks for HB models with standard envelope $Y=0.245$ and $M_{\mathrm{c}}=0.498 M_{\odot}($ dotted line $)$ are compared with tracks supposedly resulting from an evolution beyond the standard GB tip of $510^{5} \mathrm{yr}$ for $0.655 M_{\odot}$, and $10^{6} \mathrm{yr}$ for 0.63 and $0.57 M_{\odot}$. Dots indicate time intervals of $10^{7} \mathrm{yr}$

to move a structure from the red HB section to the RR Lyrae region (for $Z \sim 0.001$ ).

This is a well known fact, and has been exploited to invoke rotation as a possible "second parameter" in shaping HB morphology, since the increased pressure delays core heating (Mengel \& Gross 1976; Renzini 1977).

The question of rotation and its relevance to HB morphology has been recently investigated (Peterson et al. 1995; Behr et al. 2000a,b). An overall correspondence is found between $v \sin i$ and position on the HB, in a sense perhaps contrary to what expected. So stellar rotation rate is clearly related to the position on the $\mathrm{HB}$, but in a way not yet understood.

Independently from the causes, let us consider the consequences that a small delay in the helium flash timing can have on the HB morphology of a cluster like M 3. As mentioned before, the maximum and minimum $\mathrm{HB}$ masses for the bulk of the HB population in this cluster can be assumed to be 0.68 and $0.62 M_{\odot}(Z=0.001$, $\left.M_{\mathrm{c}}=0.498 M_{\odot}\right)$. The maximum mass, after an extra $0.510^{6}$ yr spent near the GB tip, reduces to $0.655 M_{\odot}$, while $M_{\mathrm{c}}$ increases to $0.5115 M_{\odot}$; similarly, after $10^{6} \mathrm{yr}$ one has a stellar structure of total mass $0.63 M_{\odot}$ and $M_{\mathrm{c}}=0.525$.

In Fig. 8 the evolutionary tracks of these structures are shown, together with the track derived from $0.62 M_{\odot}$ after $10^{6} \mathrm{yr}$ of extra GB evolution $\left(M=0.57 M_{\odot}, M_{\mathrm{c}}=\right.$ $0.525 M_{\odot}$ ), and the tracks (until $Y_{\mathrm{c}}=0.1$ ) of "standard" HB stars $\left(M_{\mathrm{c}}=0.498 M_{\odot}\right)$. One finds that an extra GB 
evolution of $0.510^{6} \mathrm{yr}$ is sufficient to transform the HB of M 3 into the one observed in M 2 (Lee \& Carney 1999a,b), and that an extra of $10^{6}$ yr transforms the HB morphology of M 3 into the one of M 13, exception made for the bluest members.

The extremely blue HB stars would require longer extra time, but never larger than $1.510^{6} \mathrm{yr}$, since such an extra evolution leaves a structure with about $0.006 M_{\odot}$ in the envelope. In these conditions, the subsequent evolution is the one of a "hot flasher" or flasher along the white dwarf cooling sequence, as discovered by Castellani \& Castellani (1993) and investigated also by D'Cruz et al. (1996). Such structures are found at the very blue end of foreseable HB distributions (see the quoted papers).

\section{Conclusions}

The peculiar chemical abundances in many high luminosity red giants in globular clusters apparently require an increase in their envelope helium content. It was not possible to find a confirmation of such an increase from the position of the ensuing horizontal branches: the shift from the red to the blue of the RR Lyrae variables gap requires a substantial increase in luminosity, inconsistent with observations.

The possibility is examined that the most peculiar giants belong to the second, or asymptotic, giant branch, and precisely to the phase of the helium shell flashes. Some support for the hypothesis is found in M 13, where the majority of the peculiar stars are variables of the long period, semiregular type.

An estimate is given of the consequences of a delay of the helium flash, on the basis of recent RG models: a difference in the flash timing between 0.5 and $1.510^{6} \mathrm{yr}$ can help to explain at least part of the shift from the M $3 \mathrm{HB}$ morphology to the one of $\mathrm{M} 2$, and the appearance of the extreme blue tails (M 13, NGC 6752). The many features which accompany the appearance of gaps and blue tails in HBs remain in any case unexplained.

\section{References}

Behr, B. B., Djorgovski, S. G., Cohen, J. G., et al. 2000a, ApJ, 528,849

Behr, B. B., Cohen, J. G., \& McCarthy, J. K. 2000b, ApJ, 531, L37

Bell, R. A., Hesser, J. E., \& Cannon, R. D. 1983, ApJ, 269, 580

Briley, M. M., Hesser, J. E., \& Bell, R. A. 1991, ApJ, 373, 482

Brocato, E., Castellani, V., \& Ripepi, V. 1994, AJ, 107, 622

Buonanno, R., Fusi Pecci, F., Cappellaro, E., et al. 1991, AJ, 102, 1005

Buonanno, R., Corsi, C. E., Buzzoni, A., et al. 1994, A\&A, 290, 69

Caloi, V., \& Mazzitelli, I. 1993, A\&A, 271, 139

Caloi, V., D'Antona, F., \& Mazzitelli, I. 1997, A\&A, 320, 823

Carretta, E., \& Gratton, R. G. 1997, A\&AS, 121, 95

Castellani, V., Chieffi, A., Pulone, L., \& Tornambe', A. 1985, ApJ, 296, 204

Castellani, M., \& Castellani, V. 1993, ApJ, 407, 649
Catelan, M., \& de Freitas Pachego, J. A. 1995, A\&A, 297, 345

Cavallo, R. M., Sweigart, A. V., \& Bell, R. A. 1996, ApJ, 464, L79

Cohen, J. G. 1979, ApJ, 231, 751

D'Cruz, N. L., Dorman, B., Rood, R. T., \& O'Connell, R. W. 1996, ApJ, 466, 359

Denisenkov, P. A., \& Denisenkova, S. N. 1990, AZh Pisma, 16, 642

Dorman, B., \& Rood, R. T. 1993, ApJ, 409, 387

Feast, M. W. 1989, in Evolution of Peculiar Red Giants, ed. H. R. Johnson, \& B. Zuckerman (Cambridge Univ. Press), 35

Feast, M. W. 1999, in Globular Clusters, ed. C. Martinez Roger, I. Perez Fournon, \& F. Sanchez (Cambridge Univ. Press), 251

Ferraro, F. R., Paltrinieri, B., Fusi Pecci, F., et al. 1997a, ApJ, 484, L145

Ferraro, F. R., Carretta, E., Corsi, C. E., et al. 1997b, A\&A, 320,757

Frogel, J. A. 1983, ApJ, 272, 167

Gingold, R. A. 1974, ApJ, 193, 177

Gingold, R. A. 1985, in Population II Variables, ed. F. Caputo, Mem. SAIt, 56, 169

Hill, V. 1999, A\&A, 345, 430

Johnson, J. A., \& Bolte, M. 1998, AJ, 115, 693

Kadla, Z. I., Antal, M., Zdarsky, F., \& Spasova, N. 1976, SvA, 20, 403

King, J. R., Stephens, A., Boesgaard, A. M., \& Deliyannis, C. P. 1998, AJ 115, 666

Kraft, R. P. 1994, PASP, 106, 553

Kraft, R. P., Sneden, C., Langer, G. E., \& Prosser, C. F. 1992, AJ, 104, 645

Kraft, R. P., Sneden, C., Langer, G. E., \& Shetrone, M. D. 1993, AJ, 106, 1490

Kraft, R. P., Sneden, C., Smith, G. H., et al. 1998, AJ, 115, 1500

Kurucz, R. 1993, CD-ROM 13 and CD-ROM 18

Langer, G. E., \& Hoffman, R. 1995, PASP, 107, 1177

Langer, G. E., Hoffman, R. D., \& Zaidinis, C. S. 1997, PASP, 109,244

Lee, J.-W., \& Carney, B. W. 1999a, AJ, 117, 2868

Lee, J.-W., \& Carney, B. W. 1999b, AJ, 118, 1373

Lee, Y.-W., Demarque, P., \& Zinn, R. 1994, ApJ, 423, 248

Mazzitelli, I., D’Antona, F., \& Caloi, V. 1995, A\&A, 302, 382

Mengel, J. G., \& Gross, P. G. 1976, Ap\&SS, 41, 407

Osborn, W., \& Fuenmayor, F. 1977, AJ, 82, 395

Paltrinieri, B., Ferraro, F. R., Fusi Pecci, F., \& Carretta, E. 1998, MNRAS, 293, 434

Peterson, R. C., Rood, R. T., \& Crocker, D. A. 1995, ApJ, 453, 214

Pilachowski, C. A., Sneden, C., Kraft, R. P., \& Langer, G. E. 1996, AJ, 112, 545

Renzini, A. 1977, in Advanced Stages in Stellar Evolution, 7th Advanced Course, Saas-Fee, Geneva Obs., 149

Rey, S.-C., Lee, Y.-W, Byun, Y.-I., \& Chun, M.-S. 1998, AJ, 116,1775

Russev, R. M. 1973, Perem. Zvezdi, 19, 181

Salaris, M., \& Cassisi, S. 1997, MNRAS, 289, 406

Salaris, M., \& Cassisi, S. 1998, MNRAS, 298, 166

Sandage, A. 1982, ApJ, 252, 553

Sawyer Hogg, H. B. 1973, Publ. David Dunlap Obs., 3, 6

Shetrone, M. D. 1995, BAAS, 27, 1432

Shetrone, M. D. 1996, AJ, 112, 2639 
Smith, G. H. 1987, PASP, 99, 67

Sneden, C., Kraft, P. R., Prosser, C. F., \& Langer, G. E. 1991, AJ, 102, 2001

Sneden, C., Kraft, P. R., Shetrone, M. D., et al. 1997, AJ, 114, 1964

Stetson, P. B., VandenBerg, D. A., \& Bolte, M. 1996, PASP, 108,560

Suntzeff, N. 1989, in The Abundance Spread within Globular Clusters, ed. G. Cayrel de Strobel, M. Spite, \& T. Lloyd Evans (Paris, Obs. de Paris), 71

Suntzeff, N. 1993, in The Globular Cluster-Galaxy Connection, ed. G. H. Smith, \& J. P. Brodie, PASP Conf. Ser., 48, 167 Sweigart, A. V. 1997, ApJ, 474, L23

Sweigart, A. V., \& Gross, P. G. 1976, ApJS, 32, 367

VandenBerg, D. A., Bolte, M., \& Stetson, P. B. 1990, AJ, 100, 445

Ventura, P., Zeppieri, A., Mazzitelli, I., \& D'Antona, F. 1998, A\&A, 334, 953

Walker, A. R. 1994, AJ, 108, 555

Welty, D. E. 1985, AJ, 90, 2555

Whitelock, P. A. 1986, MNRAS, 219, 525 